\title{
Exposure End Date
}

National Cancer Institute

\section{Source}

National Cancer Institute. Exposure End Date. NCI Thesaurus. Code C83138.

The stop date of the exposure. 